\title{
Existence of tripled coincidence points in ordered $b$-metric spaces and an application to a system of integral equations
}

\author{
Vahid Parvaneh ${ }^{1 *}$, Jamal Rezaei Roshan ${ }^{2}$ and Stojan Radenović ${ }^{3}$
}

\section{"Correspondence:}

vahid.parvaneh@kiau.ac.ir

'Department of Mathematics,

Gilan-E-Gharb Branch, Islamic Azad

University, Gilan-E-Gharb, Iran

Full list of author information is

available at the end of the article

\section{Springer}

\begin{abstract}
In this paper, tripled coincidence points of mappings satisfying some nonlinear contractive conditions in the framework of partially ordered $b$-metric spaces are obtained. Our results extend the results of Berinde and Borcut (Nonlinear Anal. $74: 4889-4897,2011$ ) and Borcut (Appl. Math. Comput. 218:7339-7346, 2012) from the context of ordered metric spaces to the setting of ordered $b$-metric spaces. Moreover, some examples of the main result are given. Finally, some tripled coincidence point results for mappings satisfying some contractive conditions of integral type in complete partially ordered $b$-metric spaces are deduced. Also, an application is given to support our results.
\end{abstract}

MSC: Primary $47 \mathrm{H} 10$; secondary $54 \mathrm{H} 25$

Keywords: $b$-metric space; partially ordered set; tripled fixed point

\section{Introduction and preliminaries}

Existence of coupled fixed points in partially ordered metric spaces was first investigated in 1987 by Guo and Lakshmikantham [1], and then in [2,3]. Further results in this direction under weak contraction conditions in different metric spaces were proved in, e.g., [4-6].

Recently, Berinde and Borcut [7] introduced a new concept of a tripled fixed point and obtained some tripled fixed point theorems for contractive type mappings in partially ordered metric spaces. For a survey of tripled fixed point theorems and related topics, we refer the reader to [7-11].

Definition 1.1 [7, 9] Let $(\mathcal{X}, \preceq)$ be a partially ordered set, $f: \mathcal{X}^{3} \rightarrow \mathcal{X}$ and $g: \mathcal{X} \rightarrow \mathcal{X}$.

1. An element $(x, y, z) \in \mathcal{X}^{3}$ is called a tripled fixed point of $f$ if $f(x, y, z)=x$, $f(y, x, y)=y$ and $f(z, y, x)=z$.

2. An element $(x, y, z) \in \mathcal{X}^{3}$ is called a tripled coincidence point of the mappings $f$ and $g$ if $f(x, y, z)=g x, f(y, x, y)=g y$ and $f(z, y, x)=g z$.

3. An element $(x, y, z) \in \mathcal{X}^{3}$ is called a tripled common fixed point of $f$ and $g$ if $x=g(x)=f(x, y, z), y=g(y)=f(y, x, y)$ and $z=g(z)=f(z, y, x)$.

4. We say that $f$ has the mixed $g$-monotone property if $f(x, y, z)$ is $g$-nondecreasing in $x, g$-nonincreasing in $y$ and $g$-nondecreasing in $z$, that is, if for any $x, y, z \in \mathcal{X}$,

$$
\begin{array}{ll}
x_{1}, x_{2} \in \mathcal{X}, \quad g x_{1} \preceq g x_{2} & \Rightarrow \quad f\left(x_{1}, y, z\right) \preceq f\left(x_{2}, y, z\right), \\
y_{1}, y_{2} \in \mathcal{X}, \quad g y_{1} \preceq g y_{2} & \Rightarrow \quad f\left(x, y_{1}, z\right) \succeq f\left(x, y_{2}, z\right)
\end{array}
$$

() 2013 Parvaneh et al.; licensee Springer. This is an Open Access article distributed under the terms of the Creative Commons Attribution License (http://creativecommons.org/licenses/by/2.0), which permits unrestricted use, distribution, and reproduction in any medium, provided the original work is properly cited. 
and

$$
z_{1}, z_{2} \in \mathcal{X}, \quad g z_{1} \preceq g z_{2} \quad \Rightarrow \quad f\left(x, y, z_{1}\right) \preceq f\left(x, y, z_{2}\right) .
$$

Definition 1.2 [11] Let $\mathcal{X}$ be a nonempty set. We say that the mappings $f: \mathcal{X}^{3} \rightarrow \mathcal{X}$ and $g: \mathcal{X} \rightarrow \mathcal{X}$ commute if $g(f(x, y, z))=f(g x, g y, g z)$ for all $x, y, z \in \mathcal{X}$.

In [7], Berinde and Borcut proved the following result and formulated it as Theorems 7 and 8 .

Theorem 1.3 [7] Let $(X, \preceq)$ be a partially ordered set and suppose there is a metric $d$ on $X$ such that $(X, d)$ is a complete metric space. Let $F: X^{3} \rightarrow X$ be a mapping having the mixed monotone property on $X$. Assume that there exist constants $j, k, l \in[0,1)$ with $j+k+l<1$ for which

$$
d(F(x, y, z), F(u, v, w)) \leq j d(x, u)+k d(y, v)+l d(z, w)
$$

for all $x, y, z, u, v, w \in X$ with $x \preceq u, y \succeq v$ and $z \preceq w$. Suppose either $F$ is continuous or $(X, d, \preceq)$ is regular. If there exist $x_{0}, y_{0}, z_{0} \in X$ such that $x_{0} \preceq F\left(x_{0}, y_{0}, z_{0}\right), y_{0} \succeq F\left(y_{0}, x_{0}, y_{0}\right)$ and $z_{0} \preceq F\left(z_{0}, y_{0}, x_{0}\right)$, then there exist $x, y, z \in X$ such that $F(x, y, z)=x, F(y, x, y)=y$ and $F(z, y, x)=z$.

In [10], Borcut and Berinde proved the following result and formulated it as Theorem 4.

Theorem 1.4 Let $(X, \preceq)$ be a partially ordered set and suppose there is a metric $d$ on $X$ such that $(X, d)$ is a complete metric space. Let $F: X^{3} \rightarrow X$ and $g: X \rightarrow X$ be such that $F$ has the mixed g-monotone property on $X$. Assume that there exist constants $j, k, l \in[0,1)$ with $j+k+l<1$ such that

$$
d(F(x, y, z), F(u, v, w)) \leq j d(g x, g u)+k d(g y, g v)+l d(g z, g w)
$$

for all $x, y, z, u, v, w \in X$ with $g x \preceq g u, g y \succeq g v$ and $g z \preceq g w$. Suppose that $F\left(X^{3}\right) \subseteq g(X), g$ is continuous and commutes with $F$ and also suppose either $F$ is continuous or $(X, d, \preceq)$ is regular. If there exist $x_{0}, y_{0}, z_{0} \in X$ such that $x_{0} \preceq F\left(x_{0}, y_{0}, z_{0}\right), y_{0} \succeq F\left(y_{0}, x_{0}, y_{0}\right)$ and $z_{0} \preceq$ $F\left(z_{0}, y_{0}, x_{0}\right)$, then there exist $x, y, z \in X$ such that $F(x, y, z)=g x, F(y, x, y)=g y$ and $F(z, y, x)=$ $g z$.

Notice that Theorem 1.3 follows from Theorem 1.4 by taking $g=i_{X}$ (the identity map). In [9], Borcut obtained the following.

Theorem 1.5 [9, Corollary 1] Let $(\mathcal{X}, \preceq)$ be a partially ordered set and suppose there is a metric $d$ on $\mathcal{X}$ such that $(\mathcal{X}, d)$ is a complete metric space. Let $f: \mathcal{X}^{3} \rightarrow \mathcal{X}$ and $g: \mathcal{X} \rightarrow \mathcal{X}$ be such that $f$ has the g-mixed monotone property. Assume that there exists $k \in[0,1)$ such that

$$
d(f(x, y, z), f(u, v, w)) \leq k \max \{d(g x, g u), d(g y, g v), d(g z, g w)\}
$$

for all $x, y, z, u, v, w \in \mathcal{X}$ with $g x \preceq g u, g y \succeq g v$ and $g z \preceq g w$. Suppose $f\left(\mathcal{X}^{3}\right) \subseteq g(\mathcal{X}), g$ is continuous and commutes with $f$ and also suppose either 
(a) $f$ is continuous, or

(b) $\mathcal{X}$ has the following properties:

(i) if a non-decreasing sequence $x_{n} \rightarrow x$, then $x_{n} \preceq x$ for all $n$;

(ii) if a non-increasing sequence $y_{n} \rightarrow y$, then $y_{n} \succeq y$ for all $n$.

If there exist $x_{0}, y_{0}, z_{0} \in \mathcal{X}$ such that $g x_{0} \preceq f\left(x_{0}, y_{0}, z_{0}\right), g y_{0} \succeq f\left(y_{0}, x_{0}, z_{0}\right)$ and $g z_{0} \preceq$ $f\left(z_{0}, y_{0}, x_{0}\right)$, then $f$ and $g$ have a tripled coincidence point.

The concept of a $b$-metric space was introduced by Czerwik in [12]. Since then, several papers have been published on the fixed point theory of various classes of single-valued and multi-valued operators in $b$-metric spaces (see, e.g., [13-20]).

Consistent with [12] and [20], the following definitions and results will be needed in the sequel.

Definition 1.6 [12] Let $\mathcal{X}$ be a (nonempty) set and $s \geq 1$ be a given real number. A function $d: \mathcal{X} \times \mathcal{X} \rightarrow \mathfrak{R}^{+}$is a $b$-metric if, for all $x, y, z \in \mathcal{X}$, the following conditions are satisfied:

(b $\left.\mathrm{b}_{1}\right) d(x, y)=0$ iff $x=y$,

$\left(\mathrm{b}_{2}\right) d(x, y)=d(y, x)$,

$\left(\mathrm{b}_{3}\right) d(x, z) \leq s[d(x, y)+d(y, z)]$

The pair $(\mathcal{X}, d)$ is called a $b$-metric space.

It should be noted that the class of $b$-metric spaces is effectively larger than that of metric spaces since a $b$-metric is a metric when $s=1$, and there are $b$-metric spaces which are not metric spaces. Here, we present an easy example of this kind (see also [20, p.264]).

Example 1.7 [13] Let $(\mathcal{X}, d)$ be a metric space and $\rho(x, y)=(d(x, y))^{p}$, where $p>1$ is a real number. Then $\rho$ is a $b$-metric with $s=2^{p-1}$. However, $(\mathcal{X}, \rho)$ is not necessarily a metric space.

For example, let $\mathcal{X}$ be the set of real numbers and let $d(x, y)=|x-y|$ be the usual Euclidean metric. Then $\rho(x, y)=(x-y)^{2}$ is a $b$-metric on $\Re$ with $s=2$, but is not a metric on $\Re$.

Also, the following example of a $b$-metric space was given in [19].

Example 1.8 [19] Let $\mathcal{X}$ be the set of Lebesgue measurable functions on $[0,1]$ such that

$$
\int_{0}^{1}|f(x)|^{2} d x<\infty
$$

Define $D: \mathcal{X} \times \mathcal{X} \rightarrow[0, \infty)$ by

$$
D(f, g)=\int_{0}^{1}|f(x)-g(x)|^{2} d x
$$

As $\left(\int_{0}^{1}|f(x)-g(x)|^{2} d x\right)^{1 / 2}$ is a metric on $\mathcal{X}$, then, from the previous example, $D$ is a $b$-metric on $\mathcal{X}$ with $s=2$. 
The purpose of this paper is to obtain some tripled coincidence point theorems for two mappings satisfying a $(\psi, \varphi)$-contractive condition in ordered $b$-metric spaces. Our results extend, unify and generalize the comparable results in $[7,9,10]$ from the context of ordered metric spaces to the setup of ordered $b$-metric spaces.

We also need the following definitions.

Definition 1.9 [15] Let $(\mathcal{X}, d)$ be a $b$-metric space. Then a sequence $\left\{x_{n}\right\}$ in $\mathcal{X}$ is called:

(a) $b$-convergent if there exists $x \in \mathcal{X}$ such that $d\left(x_{n}, x\right) \rightarrow 0$ as $n \rightarrow \infty$. In this case, we write $\lim _{n \rightarrow \infty} x_{n}=x$.

(b) $b$-Cauchy if $d\left(x_{n}, x_{m}\right) \rightarrow 0$ as $n, m \rightarrow \infty$.

Proposition 1.10 (See [15, Remark 2.1]) In a b-metric space $(\mathcal{X}, d)$, the following assertions hold:

$\left(\mathrm{p}_{1}\right)$ A b-convergent sequence has a unique limit.

$\left(\mathrm{p}_{2}\right)$ Each b-convergent sequence is b-Cauchy.

$\left(\mathrm{p}_{3}\right)$ In general, a b-metric is not continuous (see also an example in [16]).

Definition 1.11 [15] Let $(\mathcal{X}, d)$ and $\left(\mathcal{X}^{\prime}, d^{\prime}\right)$ be two $b$-metric spaces.

(1) The space $(\mathcal{X}, d)$ is $b$-complete if every $b$-Cauchy sequence in $\mathcal{X} b$-converges.

(2) A function $f: \mathcal{X} \rightarrow \mathcal{X}^{\prime}$ is $b$-continuous at a point $x \in \mathcal{X}$ if it is $b$-sequentially continuous at $x$, that is, whenever $\left\{x_{n}\right\}$ is $b$-convergent to $x,\left\{f\left(x_{n}\right)\right\}$ is $b$-convergent to $f(x)$.

Definition 1.12 Let $(\mathcal{X}, d)$ be a $b$-metric space. Mappings $f: \mathcal{X}^{3} \rightarrow \mathcal{X}$ and $g: \mathcal{X} \rightarrow \mathcal{X}$ are called compatible if

$$
\begin{aligned}
& \lim _{n \rightarrow \infty} d\left(g f\left(x_{n}, y_{n}, z_{n}\right), f\left(g x_{n}, g y_{n}, g z_{n}\right)\right)=0, \\
& \lim _{n \rightarrow \infty} d\left(g f\left(y_{n}, x_{n}, y_{n}\right), f\left(g y_{n}, g x_{n}, g y_{n}\right)\right)=0,
\end{aligned}
$$

and

$$
\lim _{n \rightarrow \infty} d\left(g f\left(z_{n}, y_{n}, x_{n}\right), f\left(g z_{n}, g y_{n}, g x_{n}\right)\right)=0
$$

hold whenever $\left\{x_{n}\right\},\left\{y_{n}\right\}$ and $\left\{z_{n}\right\}$ are sequences in $\mathcal{X}$ such that

$$
\begin{aligned}
& \lim _{n \rightarrow \infty} f\left(x_{n}, y_{n}, z_{n}\right)=\lim _{n \rightarrow \infty} g x_{n}, \\
& \lim _{n \rightarrow \infty} f\left(y_{n}, x_{n}, y_{n}\right)=\lim _{n \rightarrow \infty} g y_{n},
\end{aligned}
$$

and

$$
\lim _{n \rightarrow \infty} f\left(z_{n}, y_{n}, x_{n}\right)=\lim _{n \rightarrow \infty} g z_{n} .
$$

Definition 1.13 Let $\mathcal{X}$ be a nonempty set. Then $(\mathcal{X}, d, \preceq)$ is called a partially ordered $b$ metric space if $d$ is a $b$-metric on a partially ordered set $(\mathcal{X}, \preceq)$.

The space $(\mathcal{X}, d, \preceq)$ is called regular if the following conditions hold: 
(i) if a non-decreasing sequence $x_{n} \rightarrow x$, then $x_{n} \preceq x$ for all $n$,

(ii) if a non-increasing sequence $y_{n} \rightarrow y$, then $y_{n} \succeq y$ for all $n$.

The notion of an altering distance function was introduced by Khan et al. [21] as follows.

Definition 1.14 [21] The function $\psi:[0, \infty) \rightarrow[0, \infty)$ is called an altering distance function if the following properties are satisfied:

1. $\psi$ is continuous and strictly increasing.

2. $\psi(t)=0$ if and only if $t=0$.

\section{Main results}

We use the following simple lemma in proving our main results.

Lemma 2.1 Let $(\mathcal{X}, d, \preceq$ ) be an ordered $b$-metric space (with the parameter $s$ ) and let $f$ : $\mathcal{X}^{3} \rightarrow \mathcal{X}$ and $g: \mathcal{X} \rightarrow \mathcal{X}$.

(a) If a relation $\sqsubseteq$ is defined on $\mathcal{X}^{3}$ by

$$
X \sqsubseteq U \quad \Longleftrightarrow \quad x \preceq u \wedge y \succeq v \wedge z \preceq w, \quad X=(x, y, z), U=(u, v, w) \in \mathcal{X}^{3}
$$

and a mapping $D: \mathcal{X}^{3} \times \mathcal{X}^{3} \rightarrow \mathfrak{R}^{+}$is given by

$$
D(X, U)=\max \{d(x, u), d(y, v), d(z, w)\}, \quad X=(x, y, z), U=(u, v, w) \in \mathcal{X}^{3},
$$

then $\left(\mathcal{X}^{3}, D, \sqsubseteq\right)$ is an ordered b-metric space (with the same parameter $\left.s\right)$. The space $\left(\mathcal{X}^{3}, D\right)$ is b-complete iff $(\mathcal{X}, d)$ is b-complete.

(b) If the mapping $f$ has the g-mixed monotone property, then the mapping $F: \mathcal{X}^{3} \rightarrow \mathcal{X}^{3}$ given by

$$
F X=(f(x, y, z), f(y, x, y), f(z, y, x)), \quad X=(x, y, z) \in \mathcal{X}^{3}
$$

is G-nondecreasing w.r.t. $\sqsubseteq$, i.e.,

$$
G X \sqsubseteq G U \quad \Longrightarrow \quad F X \sqsubseteq F U
$$

where $G: \mathcal{X}^{3} \rightarrow \mathcal{X}^{3}$ is defined by

$$
G X=(g x, g y, g z), \quad X=(x, y, z) \in \mathcal{X}^{3} .
$$

(c) Iff is continuous from $\left(\mathcal{X}^{3}, D\right)$ to $(\mathcal{X}, d)$, then $F$ is continuous in $\left(\mathcal{X}^{3}, D\right)$.

(d) Iff and $g$ are compatible, then $F$ and $G$ are compatible.

Let $(\mathcal{X}, d, \preceq)$ be an ordered $b$-metric space, $f: \mathcal{X}^{3} \rightarrow \mathcal{X}$ and $g: \mathcal{X} \rightarrow \mathcal{X}$. In the rest of this paper, unless otherwise stated, for all $x, y, z, u, v, w \in \mathcal{X}$, let

$$
\begin{aligned}
& M_{f}(x, y, z, u, v, w) \\
& \quad=\max \{d(f(x, y, z), f(u, v, w)), d(f(y, x, y), f(v, u, v)), d(f(z, y, x), f(w, v, u))\},
\end{aligned}
$$


and

$$
M_{g}(x, y, z, u, v, w)=\max \{d(g x, g u), d(g y, g v), d(g z, g w)\}
$$

Now, the main result is presented as follows.

Theorem 2.2 Let $(\mathcal{X}, d, \preceq)$ be a partially ordered $b$-metric space with the parameter $s>1$, and let $f: \mathcal{X}^{3} \rightarrow \mathcal{X}$ and $g: \mathcal{X} \rightarrow \mathcal{X}$ be such that $f\left(\mathcal{X}^{3}\right) \subseteq g(\mathcal{X})$. Assume that

$$
\psi\left(s^{\varepsilon} M_{f}(x, y, z, u, v, w)\right) \leq \psi\left(M_{g}(x, y, z, u, v, w)\right)-\varphi\left(M_{g}(x, y, z, u, v, w)\right)
$$

for all $x, y, z, u, v, w \in \mathcal{X}$ with $g x \preceq g u, g y \succeq g v$ and $g z \preceq g w$, or $g u \preceq g x, g v \succeq g y$ and $g w \preceq g z$, where $\psi, \varphi:[0, \infty) \rightarrow[0, \infty)$ are altering distance functions and $\varepsilon>1$.

Assume also that

(1) $f$ has the mixed g-monotone property;

(2) $g$ is b-continuous and compatible with $f$.

Also, suppose that either

(a) $f$ is $b$-continuous and $(\mathcal{X}, d)$ is b-complete, or

(b) $(\mathcal{X}, d)$ is regular and $(g(\mathcal{X}), d)$ is b-complete.

If there exist $x_{0}, y_{0}, z_{0} \in \mathcal{X}$ such that $g x_{0} \preceq f\left(x_{0}, y_{0}, z_{0}\right), g y_{0} \succeq f\left(y_{0}, x_{0}, y_{0}\right)$ and $g z_{0} \preceq$ $f\left(z_{0}, y_{0}, x_{0}\right)$, then $f$ and $g$ have a tripled coincidence point in $\mathcal{X}$.

Proof Let $D$ be the $b$-metric and $\sqsubseteq$ be the partial order on $\mathcal{X}^{3}$ defined in Lemma 2.1. Also, define the mappings $F, G: \mathcal{X}^{3} \rightarrow \mathcal{X}^{3}$ by $F X=(f(x, y, z), f(y, x, y), f(z, y, x))$ and $G X=$ $(g x, g y, g z), X=(x, y, z)$ as in Lemma 2.1. Then $\left(\mathcal{X}^{3}, D, \sqsubseteq\right)$ is an ordered $b$-metric space (with the same parameter $s$ as $\mathcal{X}$ ) and $F$ is a $G$-nondecreasing mapping on it such that $F\left(\mathcal{X}^{3}\right) \subseteq$ $G\left(\mathcal{X}^{3}\right)$. Moreover, the contractive condition (1) implies that

$$
\psi\left(s^{\varepsilon} D(F X, F U)\right) \leq \psi(D(G X, G U))-\varphi(D(G X, G U))
$$

holds for all $X, U \in \mathcal{X}^{3}$ such that $G X$ and $G U$ are $\sqsubseteq$-comparable. Since $\varphi$ has non-negative values and $\psi$ is strictly increasing, (2) implies that

$$
D(F X, F U) \leq \frac{1}{s^{\varepsilon}} D(G X, G U),
$$

where $0<1 / s^{\varepsilon}<1 / s$ for all $X, U \in \mathcal{X}^{3}$ such that $G X$ and $G U$ are $\sqsubseteq$-comparable. We will prove in the next lemma that under these circumstances, it follows that $F$ and $G$ have a coincidence point $\bar{X}=(\bar{x}, \bar{y}, \bar{z}) \in \mathcal{X}^{3}$ which is obviously a tripled coincidence point of $f$ and $g$.

The following lemma is an 'ordered variant' of the basic result of Czerwik [12] (adapted for two mappings).

Lemma 2.3 Let $(\mathcal{X}, d, \preceq)$ be a partially ordered b-metric space and let $f$ and $g$ be two self-mappings on $\mathcal{X}$. Assume that there exists $\lambda \in\left[0, \frac{1}{s}\right)$ such that

$$
d(f x, f y) \leq \lambda d(g x, g y)
$$


for all $x, y \in \mathcal{X}$ with $g x \leq$ gy or $g x \succeq$ gy. Let the following conditions hold:

(i) $\quad f$ is $g$-nondecreasing with respect to $\preceq$ and $f(\mathcal{X}) \subseteq g(\mathcal{X})$;

(ii) there exists $x_{0} \in \mathcal{X}$ such that $g x_{0} \preceq f x_{0}$;

(iii) $f$ and $g$ are continuous and compatible and $(\mathcal{X}, d)$ is complete, or

(iii') $(\mathcal{X}, d, \preceq)$ is regular and one of $f(\mathcal{X})$ or $g(\mathcal{X})$ is complete.

Then $f$ and $g$ have a coincidence point in $\mathcal{X}$.

Proof Because of $f \mathcal{X} \subseteq g \mathcal{X}$ and (ii), we can define a Jungck sequence by

$$
y_{n}=f x_{n}=g x_{n+1},
$$

for all $n=0,1,2, \ldots$.

It can be proved by induction that $y_{n} \preceq y_{n+1}$ for all $n$. If $y_{n}=y_{n+1}$ for some $n$, then $x_{n+1}$ is a coincidence point of $f$ and $g$. Hence, we suppose that $y_{n} \neq y_{n+1}$ for all $n$. It can be proved in a standard way (see, e.g., [18, Lemma 3.1]) that $\left\{y_{n}\right\}$ is a Cauchy sequence.

Suppose first that (iii) holds. Then there exists

$$
\lim _{n \rightarrow \infty} f x_{n}=\lim _{n \rightarrow \infty} g x_{n}=z \in \mathcal{X}
$$

Further, since $f$ and $g$ are continuous and compatible, we get that

$$
\lim _{n \rightarrow \infty} f g x_{n}=f z, \quad \lim _{n \rightarrow \infty} g f x_{n}=g z
$$

and

$$
\lim _{n \rightarrow \infty} d\left(f g x_{n}, g f x_{n}\right)=0
$$

We will show that $f z=g z$. Indeed, we have

$$
\begin{aligned}
d(f z, g z) & \leq s\left[d\left(f z, f g x_{n}\right)+d\left(f g x_{n}, g z\right)\right]=s d\left(f z, f g x_{n}\right)+s d\left(f g x_{n}, g z\right) \\
& \leq s d\left(f z, f g x_{n}\right)+s^{2}\left[d\left(f g x_{n}, g f x_{n}\right)+d\left(g f x_{n}, g z\right)\right] \\
& \rightarrow s \cdot 0+s^{2} \cdot 0+s^{2} \cdot 0=0
\end{aligned}
$$

as $n \rightarrow \infty$, and it follows that $f z=g z$. It means that $f$ and $g$ have a coincidence point.

In the case (iii'), it follows that

$$
\lim _{n \rightarrow \infty} f x_{n}=\lim _{n \rightarrow \infty} g x_{n}=g u
$$

for some $u \in \mathcal{X}$. Because of regularity, we have $g x_{n} \preceq g u$. Applying (4) with $x=x_{n}$ and $y=u$, we have

$$
d\left(f x_{n}, f u\right) \leq \lambda d\left(g x_{n}, g u\right) \rightarrow 0 \quad(n \rightarrow \infty) .
$$

It follows that $d\left(f x_{n}, f u\right) \rightarrow 0$ when $n \rightarrow \infty$, that is, $f x_{n} \rightarrow f u$. Hence, $f$ and $g$ have a coincidence point $u \in X$. 
Let

$$
M(x, y, z, u, v, w)=\max \{d(x, u), d(y, v), d(z, w)\} .
$$

Taking $g=i_{\mathcal{X}}$ (the identity mapping on $\mathcal{X}$ ) in Theorem 2.2, we obtain the following tripled fixed point result.

Corollary 2.4 Let $(\mathcal{X}, d, \preceq)$ be a b-complete partially ordered b-metric space and let $f$ : $\mathcal{X}^{3} \rightarrow \mathcal{X}$ be a mapping having the mixed monotone property. Assume that

$$
\psi\left(s^{\varepsilon} M_{f}(x, y, z, u, v, w)\right) \leq \psi(M(x, y, z, u, v, w))-\varphi(M(x, y, z, u, v, w)),
$$

for all $x, y, z, u, v, w \in \mathcal{X}$ with $x \preceq u, y \succeq v$ and $z \preceq w$, or $u \preceq x, v \succeq y$ and $w \preceq z$, where $\psi, \varphi:[0, \infty) \rightarrow[0, \infty)$ are altering distance functions and $\varepsilon>1$.

Also, suppose that either

(a) $f$ is b-continuous, or

(b) $(\mathcal{X}, d)$ is regular.

If there exist $x_{0}, y_{0}, z_{0} \in \mathcal{X}$ such that $x_{0} \preceq f\left(x_{0}, y_{0}, z_{0}\right), y_{0} \succeq f\left(y_{0}, x_{0}, y_{0}\right)$ and $z_{0} \preceq$ $f\left(z_{0}, y_{0}, x_{0}\right)$, then $f$ has a tripled fixed point in $\mathcal{X}$.

Taking $\psi(t)=t$ and $\varphi(t)=\frac{t^{2}}{1+t}$ for all $t \in[0, \infty)$, in Corollary 2.4, we obtain the following tripled fixed point result.

Corollary 2.5 Let $(\mathcal{X}, d, \preceq)$ be a b-complete partially ordered b-metric space and let $f$ : $\mathcal{X}^{3} \rightarrow \mathcal{X}$ be a mapping having the mixed monotone property. Assume that

$$
s^{\varepsilon} M_{f}(x, y, z, u, v, w) \leq \frac{M(x, y, z, u, v, w)}{1+M(x, y, z, u, v, w)}
$$

for some $\varepsilon>1$ and all $x, y, z, u, v, w \in \mathcal{X}$ with $x \preceq u, y \succeq v$ and $z \preceq w$, or $u \preceq x, v \succeq y$ and $w \preceq z$.

Also, suppose that either

(a) $f$ is b-continuous, or

(b) $(\mathcal{X}, d)$ is regular.

If there exist $x_{0}, y_{0}, z_{0} \in \mathcal{X}$ such that $x_{0} \preceq f\left(x_{0}, y_{0}, z_{0}\right), y_{0} \succeq f\left(y_{0}, x_{0}, y_{0}\right)$ and $z_{0} \preceq$ $f\left(z_{0}, y_{0}, x_{0}\right)$, then $f$ has a tripled fixed point in $\mathcal{X}$.

Remark 2.6 1. Let in Theorem 2.2,

$$
M_{f}(x, y, z, u, v, w)=d(f(x, y, z), f(u, v, w)) .
$$

Then the contractive condition (1) reduces to the following:

$$
\psi\left(s^{\varepsilon} d(f(x, y, z), f(u, v, w))\right) \leq \psi\left(M_{g}(x, y, z, u, v, w)\right)-\varphi\left(M_{g}(x, y, z, u, v, w)\right),
$$

which appeared in [8] in the context of G-metric spaces.

Choosing the condition (1) instead of (8), brings at least two new features to the tripled fixed point theory. 
a. We obtain more general tripled coincidence point theorems, because when $f$ and $g$ satisfy condition (1), then they also satisfy (8).

b. The technique of the proof is essentially simpler than the one used in [8], that is, we need not use Lemma 14 from [8].

2. We can replace the contractive condition (1) by the following:

$$
\psi\left(s^{\varepsilon} M_{f}^{\prime}(x, y, z, u, v, w)\right) \leq \psi\left(M_{g}(x, y, z, u, v, w)\right)-\varphi\left(M_{g}(x, y, z, u, v, w)\right),
$$

where

$$
\begin{aligned}
& M_{f}^{\prime}(x, y, z, u, v, w) \\
& \quad=\frac{1}{3}[d(f(x, y, z), f(u, v, w))+d(f(y, x, y), f(v, u, v))+d(f(z, y, x), f(w, v, u))] .
\end{aligned}
$$

The following corollary can be deduced from our previously obtained results.

Corollary 2.7 Let $(\mathcal{X}, d, \preceq)$ be a partially ordered b-complete b-metric space with $s>1$. Let $f: \mathcal{X}^{3} \rightarrow \mathcal{X}$ be a mapping with the mixed monotone property such that

$$
\begin{aligned}
\psi\left(s^{\varepsilon} M_{f}(x, y, z, u, v, w)\right) \leq & \psi\left(\frac{d(x, u)+d(y, v)+d(z, w)}{3}\right) \\
& -\varphi(\max \{d(x, u), d(y, v), d(z, w)\})
\end{aligned}
$$

for some $\varepsilon>1$ and all $x, y, z, u, v, w \in \mathcal{X}$ with $x \preceq u, y \succeq v$ and $z \preceq w$, or $u \preceq x, v \succeq y$ and $w \preceq z$. Also, suppose that either

(a) $f$ is b-continuous, or

(b) $(\mathcal{X}, d, \preceq)$ is regular.

If there exist $x_{0}, y_{0}, z_{0} \in \mathcal{X}$ such that $x_{0} \preceq f\left(x_{0}, y_{0}, z_{0}\right), y_{0} \succeq f\left(y_{0}, x_{0}, y_{0}\right)$ and $z_{0} \preceq$ $f\left(z_{0}, y_{0}, x_{0}\right)$, then $f$ has a tripled fixed point in $\mathcal{X}$.

Proof If $f$ satisfies (10), then $f$ satisfies (6). Hence, the result follows from Corollary 2.4.

In Theorem 2.2, if we take $g=i_{\mathcal{X}}, \psi(t)=t$ and $\varphi(t)=(1-k) t$ for all $t \in[0, \infty)$, where $k \in[0,1)$, we obtain the following result.

Corollary 2.8 Let $(\mathcal{X}, d, \preceq)$ be a partially ordered $b$-complete $b$-metric space with $s>1$. Let $f: \mathcal{X}^{3} \rightarrow \mathcal{X}$ be a mapping having the mixed monotone property and

$$
\begin{aligned}
& \max \{d(f(x, y, z), f(u, v, w)), d(f(y, x, y), f(v, u, v)), d(f(z, y, x), f(w, v, u))\} \\
& \quad \leq \frac{k}{s^{\varepsilon}} \max \{d(x, u), d(y, v), d(z, w)\},
\end{aligned}
$$

for some $k \in[0,1), \varepsilon>1$ and all $x, y, z, u, v, w \in \mathcal{X}$ with $x \preceq u, y \succeq v$ and $z \preceq w$, or $u \preceq x$, $v \succeq y$ and $w \preceq z$. Also, suppose that either

(a) $f$ is b-continuous, or

(b) $(\mathcal{X}, d, \preceq)$ is regular. 
If there exist $x_{0}, y_{0}, z_{0} \in \mathcal{X}$ such that $x_{0} \preceq f\left(x_{0}, y_{0}, z_{0}\right), y_{0} \succeq f\left(y_{0}, x_{0}, y_{0}\right)$ and $z_{0} \preceq$ $f\left(z_{0}, y_{0}, x_{0}\right)$, then $f$ has a tripled fixed point in $\mathcal{X}$.

Corollary 2.9 Let $(\mathcal{X}, d, \preceq)$ be a partially ordered $b$-complete $b$-metric space with $s>1$. Let $f: \mathcal{X}^{3} \rightarrow \mathcal{X}$ be a mapping with the mixed monotone property such that

$$
\begin{aligned}
& \max \{d(f(x, y, z), f(u, v, w)), d(f(y, x, y), f(v, u, v)), d(f(z, y, x), f(w, v, u))\} \\
& \quad \leq \frac{k}{3 s^{\varepsilon}}[d(x, u)+d(y, v)+d(z, w)]
\end{aligned}
$$

for some $k \in[0,1), \varepsilon>1$ and all $x, y, z, u, v, w \in \mathcal{X}$ with $x \preceq u, y \succeq v$ and $z \preceq w$, or $u \preceq x$, $v \succeq y$ and $w \preceq z$. Also, suppose that either

(a) $f$ is b-continuous, or

(b) $(\mathcal{X}, d)$ is regular.

If there exist $x_{0}, y_{0}, z_{0} \in \mathcal{X}$ such that $x_{0} \preceq f\left(x_{0}, y_{0}, z_{0}\right), y_{0} \succeq f\left(y_{0}, x_{0}, y_{0}\right)$ and $z_{0} \preceq$ $f\left(z_{0}, y_{0}, x_{0}\right)$, then $f$ has a tripled fixed point in $\mathcal{X}$.

Proof If $f$ satisfies (11), then $f$ satisfies the contractive condition of Corollary 2.8.

In the following theorem, we give a sufficient condition for the uniqueness of the common tripled fixed point (see also $[7,8,11]$ ).

Theorem 2.10 In addition to the hypotheses of Theorem 2.2, suppose that $f$ and $g$ commute and that for all $(x, y, z)$ and $\left(x^{*}, y^{*}, z^{*}\right) \in \mathcal{X}^{3}$, there exists $(u, v, w) \in \mathcal{X}^{3}$ such that $(f(u, v, w), f(v, u, v), f(w, v, u))$ is comparable with $(f(x, y, z), f(y, x, y), f(z, y, x))$ and $\left(f\left(x^{*}, y^{*}, z^{*}\right), f\left(y^{*}, x^{*}, y^{*}\right), f\left(z^{*}, y^{*}, x^{*}\right)\right)$. Then $f$ and $g$ have a unique common tripled fixed point.

Proof We shall use the notation as in the proof of Theorem 2.2. It was proved in this theorem that the set of tripled coincidence points of $f$ and $g$, i.e., the set of coincidence points of $F$ and $G$ in $\mathcal{X}^{3}$, is nonempty. We shall show that if $X$ and $X^{*}$ are coincidence points of $F$ and $G$, that is, $G X=F X$ and $G X^{*}=F X^{*}$, then $G X=G X^{*}$.

Choose an element $U=(u, v, w) \in \mathcal{X}^{3}$ such that $F U=(f(u, v, w), f(v, u, v), f(w, v, u))$ is comparable with $F X$ and $F X^{*}$. Let $U_{0}=U$ and choose $U_{1} \in \mathcal{X}^{3}$ so that $G U_{1}=F U_{0}$. Then we can inductively define a sequence $\left\{G U_{n}\right\}$ such that $G U_{n+1}=F U_{n}$. Since $G X$ and $G U_{1}$ are $\sqsubseteq$-comparable, we may assume that $G X \sqsubseteq G U_{1}$. Using the mathematical induction, it is easy to prove that $G X \sqsubseteq G U_{n}$ for all $n \geq 1$. Applying (1), one obtains that

$$
\begin{aligned}
\psi\left(s^{\varepsilon} D\left(G X, G U_{n+1}\right)\right) & =\psi\left(s^{\varepsilon} D\left(F X, F U_{n}\right)\right) \\
& \leq \psi\left(D\left(G X, G U_{n}\right)\right)-\varphi\left(D\left(G X, G U_{n}\right)\right) \\
& \leq \psi\left(D\left(G X, G U_{n}\right)\right) .
\end{aligned}
$$

From the properties of $\psi$, we deduce that the sequence $\left\{D\left(G X, G U_{n}\right)\right\}$ is non-increasing. Hence, if we proceed as in Theorem 2.2, we can show that

$$
\lim _{n \rightarrow \infty} D\left(G X, G U_{n}\right)=0,
$$

that is, $\left\{G U_{n}\right\}$ is $b$-convergent to $G X$. 
Similarly, we can show that $\left\{G U_{n}\right\}$ is $b$-convergent to $G X^{*}$. Since the limit is unique, it follows that $G X=G X^{*}$.

Since $G X=F X$, by commutativity of $f$ and $g$, we have $G G X=G F X=F G X$. Let $G X=A$. Then $G A=F A$. Thus, $A$ is another coincidence point of $f$ and $g$. Then $A=G X=G A$. Therefore, $A=(a, b, c)$ is a tripled common fixed point of $f$ and $g$.

To prove the uniqueness, assume that $P$ is another common fixed point of $F$ and $G$. Then $P=G P=F P$ and also $G P=G A$. Thus, $P=G P=G A=A$. Hence, the tripled common fixed point is unique.

\section{Examples}

The following examples support our results.

Example 3.1 Let $\mathcal{X}=(-\infty, \infty)$ be endowed with the usual ordering and the complete $b$-metric $d(x, y)=(x-y)^{2}$, where $s=2$. Define $f: \mathcal{X}^{3} \rightarrow \mathcal{X}$ and $g: \mathcal{X} \rightarrow \mathcal{X}$ as

$$
f(x, y, z)=\frac{1}{3 \sqrt{3}}(x-y+z), \quad g(x)=x \sqrt{3} .
$$

Let $\psi, \varphi:[0, \infty) \rightarrow[0, \infty)$ be defined by $\psi(t)=\sqrt{t}$ and $\varphi(t)= \begin{cases}\frac{t}{3}, & t \leq 1, \\ \frac{\sqrt{t}}{3}, & t>1\end{cases}$

Now, we have

$$
\begin{aligned}
\psi\left(s^{2} d(f(x, y, z), f(u, v, w))\right)= & \sqrt{\frac{4}{27}[(x-y+z)-(u-v+w)]^{2}} \\
= & \sqrt{\frac{4}{81}[(x \sqrt{3}-u \sqrt{3})+(v \sqrt{3}-y \sqrt{3})+(z \sqrt{3}-w \sqrt{3})]^{2}} \\
\leq & \sqrt{\frac{4}{27}\left[(x \sqrt{3}-u \sqrt{3})^{2}+(y \sqrt{3}-v \sqrt{3})^{2}+(z \sqrt{3}-w \sqrt{3})^{2}\right]} \\
\leq & \frac{2}{3} \sqrt{\max \left\{(x \sqrt{3}-u \sqrt{3})^{2},(y \sqrt{3}-v \sqrt{3})^{2},(z \sqrt{3}-w \sqrt{3})^{2}\right\}} \\
= & \psi(\max \{d(g x, g u), d(g y, g v), d(g z, g w)\}) \\
& -\varphi(\max \{d(g x, g u), d(g y, g v), d(g z, g w)\}) .
\end{aligned}
$$

Analogously, we can show that

$$
\begin{aligned}
\psi\left(s^{2} d(f(y, x, y), f(v, u, v))\right) \leq & \psi(\max \{d(g x, g u), d(g y, g v), d(g z, g w)\}) \\
& -\varphi(\max \{d(g x, g u), d(g y, g v), d(g z, g w)\})
\end{aligned}
$$

and

$$
\begin{aligned}
\psi\left(s^{2} d(f(z, y, x), f(w, v, u))\right) \leq & \psi(\max \{d(g x, g u), d(g y, g v), d(g z, g w)\}) \\
& -\varphi(\max \{d(g x, g u), d(g y, g v), d(g z, g w)\}) .
\end{aligned}
$$

Thus,

$$
\psi\left(s^{2} M_{f}(x, y, z, u, v, w)\right) \leq \psi\left(M_{g}(x, y, z, u, v, w)\right)-\varphi\left(M_{g}(x, y, z, u, v, w)\right) .
$$


Hence, all of the conditions of Theorem 2.2 are satisfied (with $\varepsilon=2)$. Moreover, $(0,0,0)$ is a tripled coincidence point of $f$ and $g$.

Example 3.2 Let $\mathcal{X}=\Re$ be endowed with the usual order and the $b$-metric $d(x, y)=(x-$ $y)^{2}$ with $s=2$. Consider the mapping $f: \mathcal{X}^{3} \rightarrow \mathcal{X}$ given by

$$
f(x, y, z)=\frac{x-y+z}{40}
$$

and functions $\psi, \varphi:[0,+\infty) \rightarrow[0,+\infty)$ defined as $\psi(t)=t$ and $\varphi(t)=\frac{391}{400} t$. Take $\varepsilon=2$ in Corollary 2.4. The contractive condition (6) is satisfied since

$$
\begin{aligned}
\psi & \left(s^{2} d(f(x, y, z), f(u, v, w))\right) \\
& =4\left(\frac{x-y+z}{40}-\frac{u-v+w}{40}\right)^{2} \\
& =\frac{1}{400}[(x-u)+(v-y)+(z-w)]^{2} \leq \frac{3}{400}\left[(x-u)^{2}+(y-v)^{2}+(z-w)^{2}\right] \\
& \leq \frac{9}{400} \max \left\{(x-u)^{2},(y-v)^{2},(z-w)^{2}\right\} \\
& =\psi(\max \{d(x, u), d(y, v), d(z, w)\})-\varphi(\max \{d(x, u), d(y, v), d(z, w)\}) .
\end{aligned}
$$

It follows that $f$ has a tripled fixed point (which is $(0,0,0)$ ).

Note that if instead of the $b$-metric $d$ we try to use the standard metric $\rho(x, y)=|x-y|$ (with all other data unchanged), the conclusion cannot be obtained. Indeed, the inequality

$$
\begin{aligned}
\psi(\rho(f(x, y, z), f(u, v, w))) \leq & \psi(\max \{\rho(x, u), \rho(y, v), \rho(z, w)\}) \\
& -\varphi(\max \{\rho(x, u), \rho(y, v), \rho(z, w)\})
\end{aligned}
$$

does not hold since for $x=1, y=z=u=v=w=0$ it reduces to $\frac{1}{40} \leq \frac{9}{400}$.

Example 3.3 Let $\mathcal{X}=\{(a, 0, a): a \in[0,+\infty)\} \cup\{(0, a, 0): a \in[0,+\infty)\} \subset R^{3}$ with the order $\preceq$ be defined as

$$
\left(a_{1}, b_{1}, c_{1}\right) \preceq\left(a_{2}, b_{2}, c_{2}\right) \quad \Longleftrightarrow \quad a_{1} \leq a_{2}, \quad b_{1} \leq b_{2}, \quad c_{1} \leq c_{2} .
$$

Let $d$ be given as

$$
d(x, y)=\max \left\{\left|a_{1}-a_{2}\right|^{2},\left|b_{1}-b_{2}\right|^{2},\left|c_{1}-c_{2}\right|^{2}\right\},
$$

where $x=\left(a_{1}, b_{1}, c_{1}\right)$ and $y=\left(a_{2}, b_{2}, c_{2}\right)$. Clearly, $(\mathcal{X}, d)$ is a complete $b$-metric space with $s=2$.

Let $g: \mathcal{X} \rightarrow \mathcal{X}$ and $f: \mathcal{X}^{3} \rightarrow \mathcal{X}$ be defined as follows:

$$
f(x, y, z)=x,
$$

and $g(a, 0, a)=(0, a, 0)$ and $g(0, a, 0)=(a, 0, a)$. 
Let $\psi, \varphi:[0, \infty) \rightarrow[0, \infty)$ be two arbitrary altering distance functions.

According to the order defined on $\mathcal{X}$ and the definition of $g$, we see that for any element $x \in \mathcal{X}, g x$ is comparable only with itself.

By a careful computation, it is easy to see that all of the conditions of Theorem 2.2 (case (a)) are satisfied. Finally, Theorem 2.2 guarantees the existence of a tripled coincidence point for $f$ and $g$, i.e., the point $((0,0,0),(0,0,0),(0,0,0))$.

\section{Applications}

In this section, we obtain some tripled coincidence point theorems for a mapping satisfying a contractive condition of integral type in a complete ordered $b$-metric space.

We denote by $\Lambda$ the set of all functions $\mu:[0,+\infty) \rightarrow[0,+\infty)$ verifying the following conditions:

(I) $\mu$ is a positive Lebesgue integrable mapping on each compact subset of $[0,+\infty)$;

(II) for all $\varepsilon>0, \int_{0}^{\varepsilon} \mu(r) d r>0$

Corollary 4.1 Replace the contractive condition (1) of Theorem 2.2 by the following:

There exists a $\mu \in \Lambda$ such that

$$
\int_{0}^{\psi\left(s^{\varepsilon} M_{f}(x, y, z, u, v, w)\right)} \mu(r) d r \leq \int_{0}^{\psi\left(M_{g}(x, y, z, u, v, w)\right)} \mu(r) d r-\int_{0}^{\varphi\left(M_{g}(x, y, z, u, v, v)\right)} \mu(r) d r .
$$

Let the other conditions of Theorem 2.2 be satisfied. Then $f$ and $g$ have a tripled coincidence point.

Proof Consider the function $\Gamma(x)=\int_{0}^{x} \mu(r) d r$. Then (13) becomes

$$
\Gamma\left(\psi\left(s^{\varepsilon} M_{f}(x, y, z, u, v, w)\right)\right) \leq \Gamma\left(\psi\left(M_{g}(x, y, z, u, v, w)\right)\right)-\Gamma\left(\varphi\left(M_{g}(x, y, z, u, v, w)\right)\right) .
$$

Taking $\psi_{1}=\Gamma \circ \psi$ and $\varphi_{1}=\Gamma \circ \varphi$ and applying Theorem 2.2, we obtain the proof (it is easy to verify that $\psi_{1}$ and $\varphi_{1}$ are altering distance functions).

Corollary 4.2 Substitute the contractive condition (1) of Theorem 2.2 by the following:

There exists a $\mu \in \Lambda$ such that

$$
\begin{aligned}
& \psi\left(\int_{0}^{s^{\varepsilon} M_{f}(x, y, z, u, v, w)} \mu(r) d r\right) \\
& \quad \leq \psi\left(\int_{0}^{M_{g}(x, y, z, u, v, w)} \mu(r) d r\right)-\varphi\left(\int_{0}^{M_{g}(x, y, z, u, v, w)} \mu(r) d r\right) .
\end{aligned}
$$

Let the other conditions of Theorem 2.2 be satisfied. Then $f$ and $g$ have a tripled coincidence point.

Proof Again, as in Corollary 4.1, define the function $\Gamma(x)=\int_{0}^{x} \phi(r) d r$. Then (14) reduces to

$$
\psi\left(\Gamma\left(s^{\varepsilon} M_{f}(x, y, z, u, v, w)\right)\right) \leq \psi\left(\Gamma\left(M_{g}(x, y, z, u, v, w)\right)\right)-\varphi\left(\Gamma\left(M_{g}(x, y, z, u, v, w)\right)\right) .
$$

Now, if we define $\psi_{1}=\psi \circ \Gamma$ and $\varphi_{1}=\varphi \circ \Gamma$ and apply Theorem 2.2, then the proof is completed. 
Corollary 4.3 Replace the contractive condition (1) of Theorem 2.2 by the following:

There exists a $\mu \in \Lambda$ such that

$$
\begin{aligned}
& \psi_{1}\left(\int_{0}^{\psi_{2}\left(s^{\varepsilon} M_{f}(x, y, z, u, v, w)\right)} \mu(r) d r\right) \\
& \quad \leq \psi_{1}\left(\int_{0}^{\psi_{2}\left(M_{g}(x, y, z, u, v, w)\right)} \mu(r) d r\right)-\varphi_{1}\left(\int_{0}^{\varphi_{2}\left(M_{g}(x, y, z, u, v, w)\right)} \mu(r) d r\right)
\end{aligned}
$$

for altering distance functions $\psi_{1}, \psi_{2}, \varphi_{1}$ and $\varphi_{2}$. If the other conditions of Theorem 2.2 are satisfied, then $f$ and $g$ have a tripled coincidence point.

Similar to [22], let $N$ be a fixed positive integer. Let $\left\{\mu_{i}\right\}_{1 \leq i \leq N}$ be a family of $N$ functions which belong to $\Lambda$. For all $t \geq 0$, we define

$$
\begin{aligned}
& I_{1}(t)=\int_{0}^{t} \mu_{1}(r) d r, \\
& I_{2}(t)=\int_{0}^{I_{1} t} \mu_{2}(r) d r=\int_{0}^{\int_{0}^{t} \mu_{1}(r) d r} \mu_{2}(r) d r, \\
& I_{3}(t)=\int_{0}^{I_{2} t} \mu_{3}(r) d r=\int_{0}^{\int_{0}^{\int_{0}^{t} \mu_{1}(r) d r} \mu_{2}(r) d r} \mu_{3}(r) d r, \\
& I_{N}(t)=\int_{0}^{I_{(N-1)} t} \mu_{N}(r) d r .
\end{aligned}
$$

We have the following result.

Corollary 4.4 Replace the inequality (1) of Theorem 2.2 by the following condition:

$$
\psi\left(I_{N}\left(s^{\varepsilon} M_{f}(x, y, z, u, v, w)\right)\right) \leq \psi\left(I_{N}\left(M_{g}(x, y, z, u, v, w)\right)\right)-\varphi\left(I_{N}\left(M_{g}(x, y, z, u, v, w)\right)\right) .
$$

Let the other conditions of Theorem 2.2 be satisfied. Then $f$ and $g$ have a tripled coincidence point.

Proof Consider $\hat{\Psi}=\psi \circ I_{N}$ and $\hat{\Phi}=\varphi \circ I_{N}$. Then the above inequality becomes

$$
\hat{\Psi}\left(s^{\varepsilon} M_{f}(x, y, z, u, v, w)\right) \leq \hat{\Psi}\left(M_{g}(x, y, z, u, v, w)\right)-\hat{\Phi}\left(M_{g}(x, y, z, u, v, w)\right) .
$$

Applying Theorem 2.2, we obtain the desired result (it is easy to verify that $\hat{\Psi}$ and $\hat{\Phi}$ are altering distance functions).

Another consequence of the main theorem is the following result.

Corollary 4.5 Substitute the contractive condition (1) of Theorem 2.2 by the following: 
There exist $\mu_{1}, \mu_{2} \in \Lambda$ such that

$$
\int_{0}^{s^{\varepsilon} M_{f}(x, y, z, u, v, w)} \mu_{1}(r) d r \leq \int_{0}^{M_{g}(x, y, z, u, v, w)} \mu_{1}(r) d r-\int_{0}^{M_{g}(x, y, z, u, v, w)} \mu_{2}(r) d r .
$$

Let the other conditions of Theorem 2.2 be satisfied. Then $f$ and $g$ have a tripled coincidence point.

Proof It is clear that the function $t \rightarrow \int_{0}^{t} \mu_{i}(r) d r$ is an altering distance function.

\section{Existence of a solution for a system of integral equations}

Motivated by the work in [8], we study the existence of solutions for a system of nonlinear integral equations using the results proved in the previous sections.

Consider the integral equations in the following system.

$$
\begin{aligned}
& x(t)=P(t)+\int_{0}^{T} S(t, r)[f(r, x(r))+k(r, y(r))+h(r, z(r))] d r, \\
& y(t)=P(t)+\int_{0}^{T} S(t, r)[f(r, y(r))+k(r, x(r))+h(r, y(r))] d r, \\
& z(t)=P(t)+\int_{0}^{T} S(t, r)[f(r, z(r))+k(r, y(r))+h(r, x(r))] d r .
\end{aligned}
$$

We will consider the system (17) under the following assumptions:

(i) $f, k, h:[0, T] \times \Re \rightarrow \Re$ are continuous;

(ii) $P:[0, T] \rightarrow \Re$ is continuous;

(iii) $S:[0, T] \times \Re \rightarrow[0, \infty)$ is continuous;

(iv) there exists $q>0$ such that for all $x, y \in \Re$,

$$
\begin{aligned}
& 0 \leq f(r, y)-f(r, x) \leq q(y-x) \\
& 0 \leq k(r, x)-k(r, y) \leq q(y-x)
\end{aligned}
$$

and

$$
0 \leq h(r, y)-h(r, x) \leq q(y-x)
$$

(v)

$$
2^{4 p-4} 3 q^{p} \max _{t \in[0, T]}\left(\int_{0}^{T}|S(t, r)| d r\right)^{p}<1
$$

(vi) there exist continuous functions $\alpha, \beta, \gamma:[0, T] \rightarrow \Re$ such that

$$
\begin{aligned}
& \alpha(t) \leq P(t)+\int_{0}^{T} S(t, r)[f(r, \alpha(r))+k(r, \beta(r))+h(r, \gamma(r))] d r \\
& \beta(t) \geq P(t)+\int_{0}^{T} S(t, r)[f(r, \beta(r))+k(r, \alpha(r))+h(r, \beta(r))] d r
\end{aligned}
$$


and

$$
\gamma(t) \leq P(t)+\int_{0}^{T} S(t, r)[f(r, \gamma(r))+k(r, \beta(r))+h(r, \alpha(r))] d r
$$

We consider the space $\mathcal{X}=C([0, T], \Re)$ of continuous functions defined on $[0, T]$ endowed with the $b$-metric given by

$$
d(u, v)=\max _{t \in[0, T]}|u(t)-v(t)|^{p}
$$

for all $u, v \in \mathcal{X}$, where $s=2^{p-1}$ and $p \geq 1$. We endow $\mathcal{X}$ with the partial order $\preceq$ given by

$$
x \preceq y \quad \Longleftrightarrow \quad x(t) \leq y(t)
$$

for all $t \in[0, T]$.

It is known that $(\mathcal{X}, d, \preceq)$ is regular $[23]$.

Our result is the following.

Theorem 5.1 Under assumptions (i)-(vi), the system (17) has a solution in $\mathcal{X}^{3}$, where $\mathcal{X}=$ $C([0, T], \Re)$.

Proof As in [8], we consider the operators $F: \mathcal{X}^{3} \rightarrow \mathcal{X}$ and $g: \mathcal{X} \rightarrow \mathcal{X}$ defined by

$$
F\left(x_{1}, x_{2}, x_{3}\right)(t)=P(t)+\int_{0}^{T} S(t, r)\left[f\left(r, x_{1}(r)\right)+k\left(r, x_{2}(r)\right)+h\left(r, x_{3}(r)\right)\right] d r
$$

and $g(x)=x$ for all $t \in[0, T], x_{1}, x_{2}, x_{3}, x \in \mathcal{X}$.

$F$ has the mixed monotone property (see [8, Theorem 25]).

Let $x, y, z, u, v, w \in \mathcal{X}$, with $x \succeq u, y \preceq v$ and $z \succeq w$. Since $F$ has the mixed monotone property, we have

$$
F(u, v, w) \preceq F(x, y, z) .
$$

On the other hand,

$$
d(F(x, y, z), F(u, v, w))=\max _{t \in[0, T]}|F(x, y, z)(t)-F(u, v, w)(t)|^{p} .
$$

Note that for all $t \in[0, T]$, from (iv) and the fact that for all $a, b, c \geq 0,(a+b+c)^{p} \leq$ $2^{2 p-2} a^{p}+2^{2 p-2} b^{p}+2^{p-1} c^{p}$, we have

$$
\begin{aligned}
& (|F(x, y, z)(t)-F(u, v, w)(t)|)^{p} \\
& =\mid \int_{0}^{T} S(t, r)[f(r, x(r))-f(r, u(r))] d r \\
& \quad+\int_{0}^{T} S(t, r)[k(r, y(r))-k(r, v(r))] d r \\
& \quad+\left.\int_{0}^{T} S(t, r)[h(r, z(r))-h(r, w(r))] d r\right|^{p}
\end{aligned}
$$




$$
\begin{aligned}
& \leq\left(\left|\int_{0}^{T} S(t, r)[f(r, x(r))-f(r, u(r))] d r\right|\right. \\
& +\left|\int_{0}^{T} S(t, r)[k(r, y(r))-k(r, v(r))] d r\right| \\
& \left.+\left|\int_{0}^{T} S(t, r)[h(r, z(r))-h(r, w(r))] d r\right|\right)^{p} \\
& \leq\left(2^{2 p-2}\left|\int_{0}^{T} S(t, r)[f(r, x(r))-f(r, u(r))] d r\right|^{p}\right. \\
& +2^{2 p-2}\left|\int_{0}^{T} S(t, r)[k(r, y(r))-k(r, v(r))] d r\right|^{p} \\
& \left.+2^{p-1}\left|\int_{0}^{T} S(t, r)[h(r, z(r))-h(r, w(r))] d r\right|^{p}\right) \\
& \leq 2^{2 p-2}\left[\left(\int_{0}^{T}|S(t, r)[f(r, x(r))-f(r, u(r))]| d r\right)^{p}\right. \\
& +\left(\int_{0}^{T}|S(t, r)[k(r, y(r))-k(r, v(r))]| d r\right)^{p} \\
& \left.+\left(\int_{0}^{T}|S(t, r)[h(r, y(r))-h(r, v(r))]| d r\right)^{p}\right] \\
& \leq 2^{2 p-2} q^{p}\left[\left(\max _{r \in[0, T]}|x(r)-u(r)|\right)^{p}+\left(\max _{r \in[0, T]}|y(r)-v(r)|\right)^{p}\right. \\
& \left.+\left(\max _{r \in[0, T]}|z(r)-w(r)|\right)^{p}\right]\left(\int_{0}^{T}|S(t, r)| d r\right)^{p} \\
& =2^{2 p-2} q^{p}\left[\max _{r \in[0, T]}|x(r)-u(r)|^{p}+\max _{r \in[0, T]}|y(r)-v(r)|^{p}\right. \\
& \left.+\max _{r \in[0, T]}|z(r)-w(r)|^{p}\right]\left(\int_{0}^{T}|S(t, r)| d r\right)^{p} .
\end{aligned}
$$

Thus,

$$
\begin{aligned}
& \max _{t \in[0, T]}(F(x, y, z)(t)-F(u, v, w)(t))^{p} \\
& \quad \leq 2^{2 p-2} q^{p}[d(x, u)+d(y, v)+d(z, w)] \max _{t \in[0, T]}\left(\int_{0}^{T}|S(t, r)| d r\right)^{p} \\
& \quad \leq 2^{2 p-2} 3 q^{p} \max \{d(x, u), d(y, v), d(z, w)\} \max _{t \in[0, T]}\left(\int_{0}^{T}|S(t, r)| d r\right)^{p}
\end{aligned}
$$

Repeating this idea, using the definition of the $b$-metric $d$, we get

$$
\begin{aligned}
& \max _{t \in[0, T]}(F(y, x, y)(t)-F(v, u, v)(t))^{p} \\
& \quad \leq 2^{2 p-2} q^{p}[d(y, v)+d(x, u)+d(y, v)] \max _{t \in[0, T]}\left(\int_{0}^{T}|S(t, r)| d r\right)^{p} \\
& \quad \leq 2^{2 p-2} 3 q^{p} \max \{d(y, v), d(x, u)\} \max _{t \in[0, T]}\left(\int_{0}^{T}|S(t, r)| d r\right)^{p} \\
& \quad \leq 2^{2 p-2} 3 q^{p} \max \{d(x, u), d(y, v), d(z, w)\} \max _{t \in[0, T]}\left(\int_{0}^{T}|S(t, r)| d r\right)^{p}
\end{aligned}
$$


and

$$
\begin{aligned}
& \max _{t \in[0, T]}(F(z, y, x)(t)-F(w, v, u)(t))^{p} \\
& \quad \leq 2^{2 p-2} q^{p}[d(x, u)+d(y, v)+d(z, w)] \max _{t \in[0, T]}\left(\int_{0}^{T}|S(t, r)| d r\right)^{p} \\
& \quad \leq 2^{2 p-2} 3 q^{p} \max \{d(x, u), d(y, v), d(z, w)\} \max _{t \in[0, T]}\left(\int_{0}^{T}|S(t, r)| d r\right)^{p} .
\end{aligned}
$$

Hence, from the above three inequalities, we have

$$
\begin{gathered}
\max \{d(F(x, y, z), F(u, v, w)), d(F(y, x, y), F(v, u, v)), d(F(z, y, x), F(w, v, u))\} \\
\leq 2^{2 p-2} 3 q^{p} \max _{t \in[0, T]}\left(\int_{0}^{T}|S(t, r)| d r\right)^{p} \max \{d(x, u), d(y, v), d(z, w)\} \\
\leq \frac{2^{4 p-4} 3 q^{p} \max _{t \in[0, T]}\left(\int_{0}^{T}|S(t, r)| d r\right)^{p}}{2^{2 p-2}} \max \{d(x, u), d(y, v), d(z, w)\} .
\end{gathered}
$$

But from (v), we have

$$
2^{4 p-4} 3 q^{p} \max _{t \in[0, T]}\left(\int_{0}^{T}|S(t, r)| d r\right)^{p}<1 .
$$

This proves that the operator $F$ satisfies the contractive condition appearing in Corollary 2.8 (with $\varepsilon=2$ ).

Let $\alpha, \beta, \gamma$ be the functions appearing in assumption (vi). Then by (vi) we get

$$
\alpha \preceq F(\alpha, \beta, \gamma), \quad \beta \succeq F(\beta, \alpha, \beta), \quad \gamma \preceq F(\gamma, \beta, \alpha) .
$$

Applying Corollary 2.8, we deduce the existence of $x_{1}, x_{2}, x_{3} \in \mathcal{X}$ such that $x_{1}=$ $F\left(x_{1}, x_{2}, x_{3}\right), x_{2}=F\left(x_{2}, x_{1}, x_{2}\right)$ and $x_{3}=F\left(x_{3}, x_{2}, x_{1}\right)$.

\section{Competing interests}

The authors declare that they have no competing interests.

\section{Authors' contributions}

JRR, VP and SR have worked together on each section of the paper such as the literature review, results and examples. All authors read and approved the final manuscript.

\section{Author details}

'Department of Mathematics, Gilan-E-Gharb Branch, Islamic Azad University, Gilan-E-Gharb, Iran. ${ }^{2}$ Department of Mathematics, Qaemshahr Branch, Islamic Azad University, Qaemshahr, Iran. ${ }^{3}$ Faculty of Mechanical Engineering, University of Belgrade, Kraljice Marije 16, Beograd, 11120, Serbia.

\section{Acknowledgements}

The authors express their gratitude to the referees and Professor Zoran Kadelburg for their helpful suggestions which improved the presentation, in particular the proof of Theorem 2.2.

Received: 4 March 2013 Accepted: 29 April 2013 Published: 16 May 2013

\section{References}

1. Guo, D, Lakshmikantham, V: Coupled fixed points of nonlinear operators with applications. Nonlinear Anal. 11, 623-632 (1987)

2. Gnana Bhaskar, T, Lakshmikantham, V: Fixed point theorems in partially ordered metric spaces and applications. Nonlinear Anal. 65, 1379-1393 (2006) 
3. Lakshmikantham, V, Ćirić, L: Coupled fixed point theorems for nonlinear contractions in partially ordered metric spaces. Nonlinear Anal. 70(12), 4341-4349 (2009)

4. Aydi, H, Postolache, M, Shatanawi, W: Coupled fixed point results for $(\psi, \varphi)$-weakly contractive mappings in ordered G-metric spaces. Comput. Math. Appl. 63, 298-309 (2012)

5. Luong, NV, Thuan, NX: Coupled fixed points in partially ordered metric spaces and application. Nonlinear Anal. 74, 983-992 (2011)

6. Shatanawi, W: Coupled fixed point theorems in generalized metric spaces. Hacet. J. Math. Stat. 40, 441-447 (2011)

7. Berinde, V, Borcut, M: Tripled fixed point theorems for contractive type mappings in partially ordered metric spaces. Nonlinear Anal. 74, 4889-4897 (2011)

8. Aydi, H, Karapınar, E, Shatanawi, W: Tripled coincidence point results for generalized contractions in ordered generalized metric spaces. Fixed Point Theory Appl. 2012, 101 (2012)

9. Borcut, M: Tripled coincidence theorems for contractive type mappings in partially ordered metric spaces. Appl. Math. Comput. 218, 7339-7346 (2012)

10. Borcut, $M$, Berinde, $V$ : Tripled coincidence theorems for contractive type mappings in partially ordered metric spaces. Appl. Math. Comput. 218, 5929-5936 (2012)

11. Choudhury, BS, Karapınar, E, Kundu, A: Tripled coincidence point theorems for nonlinear contractions in partially ordered metric spaces. Int. J. Math. Math. Sci. 2012, Article ID 329298 (2012)

12. Czerwik, S: Contraction mappings in b-metric spaces. Acta Math. Inform. Univ. Ostrav. 1, 5-11 (1993)

13. Aghajani, A, Abbas, M, Roshan, JR: Common fixed point of generalized weak contractive mappings in partially ordered $b$-metric spaces. Math. Slovaca (in press)

14. Akkouchi, $\mathrm{M}$ : Common fixed point theorems for two selfmappings of a $b$-metric space under an implicit relation. Hacet. J. Math. Stat. 40(6), 805-810 (2011)

15. Boriceanu, M, Bota, M, Petrusel, A: Multivalued fractals in b-metric spaces. Cent. Eur. J. Math. 8(2), 367-377 (2010)

16. Hussain, N, Đorić, D, Kadelburg, Z, Radenovi ć, S: Suzuki-type fixed point results in metric type spaces. Fixed Point Theory Appl. 2012, 126 (2012)

17. Hussain, N, Shah, MH: KKM mappings in cone b-metric spaces. Comput. Math. Appl. 62, 1677-1684 (2011)

18. Jovanović, M, Kadelburg, Z, Radenović, S: Common fixed point results in metric-type spaces. Fixed Point Theory Appl. 2010, Article ID 978121 (2010). doi:10.1155/2010/978121

19. Khamsi, MA, Hussain, N: KKM mappings in metric type spaces. Nonlinear Anal. 73(9), 3123-3129 (2010)

20. Singh, SL, Prasad, B: Some coincidence theorems and stability of iterative procedures. Comput. Math. Appl. 55, 2512-2520 (2008)

21. Khan, MS, Swaleh, M, Sessa, S: Fixed point theorems by altering distances between the points. Bull. Aust. Math. Soc. 30, 1-9 (1984)

22. Nashine, HK, Samet, B: Fixed point results for mappings satisfying $(\psi, \varphi)$-weakly contractive condition in partially ordered metric spaces. Nonlinear Anal. 74, 2201-2209 (2011)

23. Nieto, JJ, Rodriguez-López, R Existence and uniqueness of fixed point in partially ordered sets and applications to ordinary differential equations. Order 22(3), 223-239 (2005)

doi:10.1186/1687-1812-2013-130

Cite this article as: Parvaneh et al.: Existence of tripled coincidence points in ordered $b$-metric spaces and an application to a system of integral equations. Fixed Point Theory and Applications 2013 2013:130.

\section{Submit your manuscript to a SpringerOpen ${ }^{\ominus}$ journal and benefit from:}

- Convenient online submission

Rigorous peer review

- Immediate publication on acceptance

- Open access: articles freely available online

- High visibility within the field

- Retaining the copyright to your article 